\title{
Resolution analysis in computational imaging with patterned illumination and bucket detection
}

\author{
A. D. Rodríguez, ${ }^{1, *}$ P. Clemente, ${ }^{2}$ E. Irles, ${ }^{1}$ E. Tajahuerce, ${ }^{1}$ and J. Lancis ${ }^{1}$ \\ ${ }^{1}$ Institut de Noves Tecnologies de la Imatge (INIT), Universitat Jaume I, Castelló 12071, Spain \\ ${ }^{2}$ Servei Central d'Instrumentació Científica (SCIC), Universitat Jaume I, Castelló 12071, Spain \\ *Corresponding author: jimeneza@uji.es \\ Received April 1, 2014; revised May 8, 2014; accepted May 21, 2014; \\ posted May 23, 2014 (Doc. ID 209268); published June 24, 2014
}

\begin{abstract}
In computational imaging by pattern projection, a sequence of microstructured light patterns codified onto a programmable spatial light modulator is used to sample an object. The patterns are used as generalized measurement modes where the object information is expressed. In this Letter, we show that the resolution of the recovered image is only limited by the numerical aperture of the projecting optics regardless of the quality of the collection optics. We provide proof-of-principle experiments where the single-pixel detection strategy outperforms the resolution achieved using a conventional optical array detector for optical imaging. It is advantageous in the presence of real-world conditions, such as optical aberrations and optical imperfections in between the sample and the sensor. We provide experimental verification of image retrieval even when an optical diffuser prevents imaging with a megapixel array camera. (c) 2014 Optical Society of America

OCIS codes: (110.0180) Microscopy; (110.1758) Computational imaging; (220.1000) Aberration compensation; (350.5730) Resolution.

http://dx.doi.org/10.1364/OL.39.003888
\end{abstract}

Despite ongoing improvements in optical array detector technologies such as charge-coupled devices (CCDs) and electron multiplying charge-coupled devices (EMCCDs) cameras, both point and line scanning methods provide the method of choice in several imaging devices as, for instance, in confocal or multiphoton microscopy. In the former, dot-by-dot illumination of the scene with a femtosecond laser pulse triggers spatially resolved multiphoton absorption, which is the key for optical sectioning. Single-pixel detection allows us to achieve low-light sensitivity as well as multidimensional capability through the use of specialized sensing.

Although systems that scan a single-element benefit from mature technology, they suffer from acquisition times linearly proportional to the spatial resolution. Significant per-pixel dwell times limit real-time performance to low resolution. A promising option is to use global illumination strategies while preserving a single-pixel "bucket" detector. To do this, the sample is obtained by imaging the scene through microstructured masks implemented onto a programmable spatial light modulator (SLM). In this approach, a single-pixel detector measures the transmitted or reflected power as the pattern generated in the SLM changes in a sequential manner. The programmed patterns are used as generalized measurement modes where the object information is expressed and the sample is recovered through algebraic optimization. This principle enables retrieval of the spatial information of an object in ghost $[1,2]$ and compressive imaging [3]. Among the applications that benefit from the potential advantages of the so-called "singlepixel cameras" are fluorescence microscopy [4]], hyperspectral imaging [5], and polarimetric imaging []], to list only a few.

The fundamental reason why the bucket detection strategy can outperform conventional optical array detection is the use of a single channel detector that simultaneously integrates all the photons transmitted through the patterned scene. As a matter of fact, incoherent measurements used in imaging with patterned illumination use half the available light on average so that they match low-light level conditions. This requires large linear dynamic range photodetectors. However, this approach has been demonstrated to work at sub-picowatt light levels by using photomultiplier tubes and Geigermode avalanche photodiodes that provide shot-noise limited detection [7]. This type of sensor is difficult to integrate in an arrayed detector.

On the other hand, a main difference between the serial scanning and global illumination approaches is related to temporal resolution. While serial excitation is mainly limited by the dwell time at a given position, that is, the time to induce a detectable response, global illumination schemes demand a short refreshing rate for the SLM, which can be of the order of tens of kilohertz if a digital micromirror device (DMD) is used as an SLM. The multiplex advantage in single-pixel cameras has been successfully employed to acquire the collection of chemical images in less than $1 \mathrm{~min}$ in multivariate hyperspectral Raman imaging []. This represents a speed advantage of the order of 100 .

Single-pixel cameras also benefit from the compressive detection strategy [9]. Most images of interest that are obtained in experiments or by numerical computation of natural phenomena are sparse. In compressive sampling (CS), it is possible to make use of this fact to recover an image from fewer measurements than those expected by the Nyquist sampling rate, often less than $10 \%$. The approach is so named because it allows image compression to be performed during measurement rather than in the post-processing stage.

In this Letter, we focus on the analysis of resolution in optical imaging with patterned illumination and bucket detection. The reasons for our research are twofold. On the one hand, speckle illumination and second-order correlation measurement have shown to provide subRayleigh limit imaging $[\underline{10}, 11]$. Imaging with patterned illumination and bucket detection is closely related to 
the above technique in the sense that the information in incoherent measurements used for compressive sensing is contained in their deviation from the mean. Also, despite the commonly held opinion that algorithmic methods for subwavelength imaging are impractical, sparsity-based subwavelength imaging has demonstrated recovery of $100 \mathrm{~nm}$ features in a sample illuminated with light of wavelength of $532 \mathrm{~nm}$ [12]. This superresolution feature works for both coherent and incoherent light. In the same direction, virtually structured detection through mathematical processing of digital images has been proposed as the dual technique of structured illumination microscopy technique [13]. On the other hand, ghost imaging has been claimed as an alternative to carry out image transmission through an aberrating medium [14]. In the same context, the possibility for image transmission through highly scattering medium is of a high practical interest [15].

Our Letter makes two specific contributions within the field of single-pixel imaging through patterned illumination. First, we perform an analysis of the optical resolution of the computational image. This resolution is shown not to be limited at all by the optical quality of the collection optics. This result is proved by using a low NA microscope objective for imaging at a CCD camera. Spatial frequencies that are not transmitted through this low quality optics are demonstrated to be present in the retrieved image through patterned illumination. Second, we experimentally demonstrate the capability of our technique to properly recover an image even when an optical diffuser is located in between the sample and the bucket detector.

As mentioned above, most images of interest are sparse in some particular basis functions. Let us suppose that an $N$-pixel image has only $S$ nonzero significant coefficients in a given basis. CS states that an $M$-random subset of coefficients of the image expressed in this basis contains enough information for image reconstruction with high accuracy [3,9]. The CS technique asserts the image can be retrieved if $M \geq S \log N$, overcoming the Nyquist sample rate and achieving a compression ratio $\mathrm{CR}=N / M$. In our experiment, a single-pixel detection system is implemented in order to measure these $M$ coefficients by projecting the object onto the random subset of functions in an iterative acquisition process. The selection of these coefficients of the unknown image can be expressed as

$$
\mathbf{y}=\mathbf{S x}
$$

where $\mathbf{x}$ is the $N$-pixel unknown image expressed in the chosen basis, $\mathbf{y}$ is the subset of $M$-coefficients measured by projection process and $\mathbf{S}$ is the sensing matrix. The underdetermined matrix relation obtained after the measurement process has an infinite number of solutions. Assuming the sparsity of the solution, CS allows us to find a unique one. In our case, the approach to solve the problem is based on the minimization of the $l_{1}$-norm of $\mathbf{x}$ subjected to the constraint given by Eq. (1), that is

$$
\min \|\mathbf{x}\|_{l_{1}} \quad \text { such that } \mathbf{y}=\mathbf{S x} \text {, }
$$

where $\|\mathbf{x}\|_{l_{1}}=\sum_{i}\left|x_{i}\right|$. A suitable algorithm is used to find the minimum that leads to the solution, namely, the

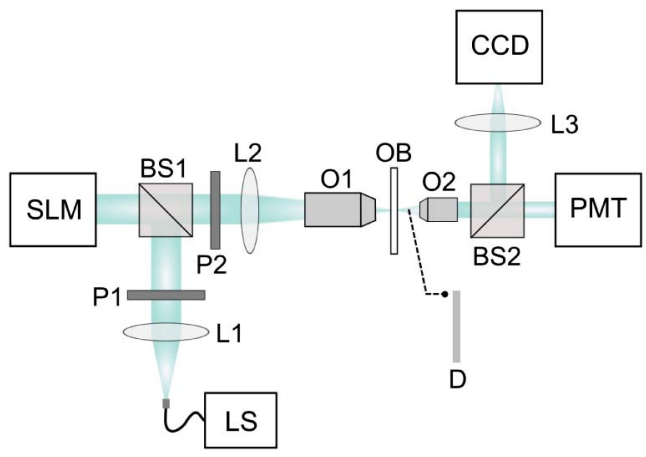

Fig. 1. Experimental setup for image resolution analysis via Hadamard illumination using a single-pixel architecture. See text for details.

retrieved image. In our experiments, Walsh-Hadamard functions are chosen as the projection basis and the l1eq-pd programming code is employed in the off-line reconstruction [16].

A Walsh-Hadamard matrix of order $n$ is a binary $n \times n$ matrix with \pm 1 entries that satisfies $H_{n}^{T} H_{n}=n I_{n}$, where $I_{n}$ is the identity matrix and $H_{n}^{T}$ denotes transposed matrix. Walsh-Hadamard matrices form an orthonormal basis with an optimum weighting design for extracting information from random noise.

The single-pixel detection system used to analyze the resolution limit is shown in Fig. 1. The optical setup can be divided into two parts: the illumination system and the collection system, located left and right of the object OB, respectively. In the illumination system, the SLM generates binary patterns modulated on amplitude sequentially. These patterns are reduced and projected onto the object through an optical lens and a microscope objective. In the collection system, a microscope objective with low NA collects the light transmitted by the object for each pattern. A photomultiplier tube (PMT) integrates the light provided by the objective and returns the value of the coefficient associated to each pattern. Finally, the image is reconstructed by applying the optimization algorithm in Eq. (2) to this set of coefficients. To compare the optical resolution of our single-pixel system with that of a conventional one, a CCD captures a conventional image of the object by using the same low NA objective. The details of the optical elements in Fig. 1 are the following. The light source (LS) is a mercury lamp (Nikon, Intensilight C-HGFI). In order to achieve quasi-monochromatic signal, the light is filtered with a color filter (part of Pasco AP-9368). After passing through a collimator lens (L1), the light illuminates the SLM (a reflective liquid crystal on silicon display, Holoeye LC-R 2500) with XGA resolution and a pixel pitch of $19 \mu \mathrm{m}$. A beam splitter (BS1) allows this SLM to work at normal incidence. Both SLM and BS1 were sandwiched between crossed polarizers (P1 and P2) to get amplitude modulation. The light patterns are projected onto the object by the objective $\mathrm{O} 1$ (Nikon LU Plan 20X/0.40 WD 13). The outgoing light is collected with the microscope objective O2 (Nikon E Plan 4X/0.10 WD 30). A second beam splitter (BS2) permits us to both observe the light projection by a CCD (Basler A102fc, pixel size $=6.45 \mu \mathrm{m} \times 6.45 \mu \mathrm{m}$ ) and capture the intensity using a PMT (PMM01, Thorlabs 
Inc.). With the aim of analyzing the optical resolution in both the conventional and the single-pixel system, a USAF test chart (OB, USAF $19512^{\prime \prime} \times 2^{\prime \prime}$, Edmund Optics) is employed as the object.

The resolution of the illumination system is limited by diffraction by the aperture of $\mathrm{O} 1$ to $0.8 \mu \mathrm{m}$, while the resolution is limited geometrically by the pixel size of the SLM in the sample plane after the reduction produced by L2 and $\mathrm{O} 1$ to $1.68 \mu \mathrm{m}$. As the recording system is based on detecting fluctuations of the integrated intensity, instead of resolving the spatial information, the collecting system has no influence on the resolution. In this sense, the system resembles to some extent Type I scanning optical microscopes but with wide-field illumination [17]. Note that $\mathrm{O} 2$ is only necessary to form the image on the CCD. Indeed if O2 were removed and the PMT placed directly behind the sample, the imaging resolution would remain the same.

The experimental results are shown in Fig. 2. Figure 2(a) shows the portion of the USAF test imaged by our optical system. Only the smallest groups of the test, 6 and 7, are used to bound the resolution of the systems. The optical system is designed in such a way that the resolution is not restricted geometrically by the size of the pixels of the CCD. Therefore, in principle, the theoretical resolution of the conventional system is
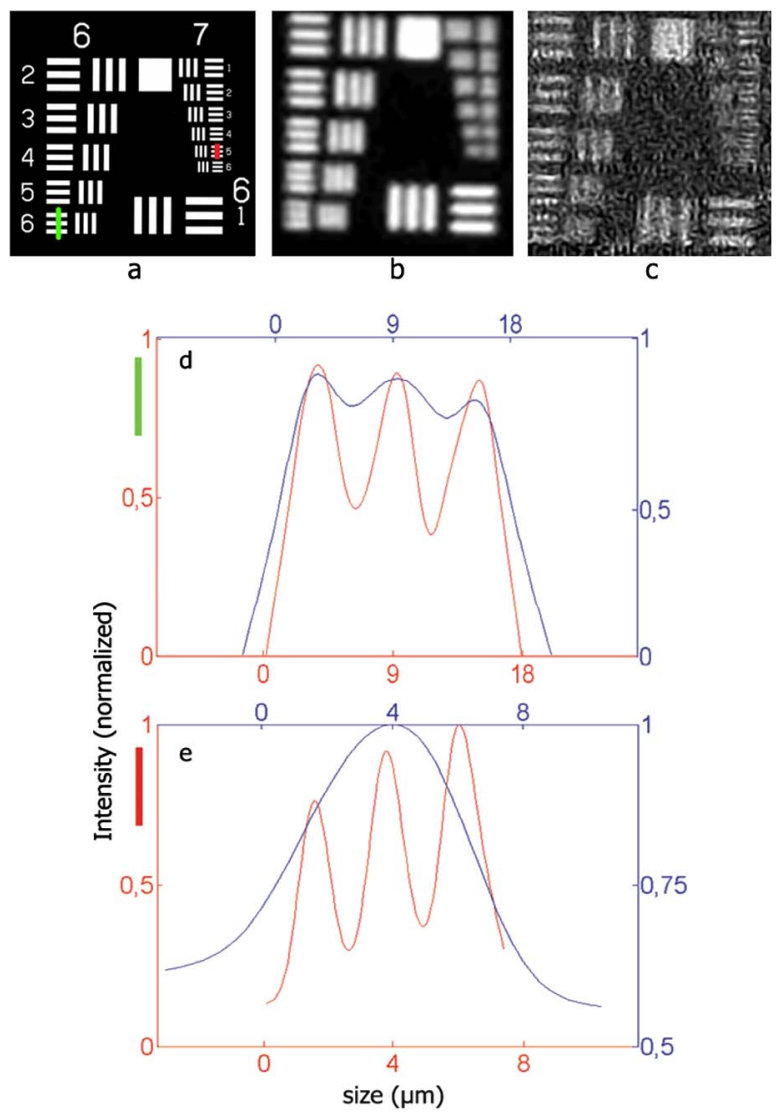

Fig. 2. Experimental results for image resolution analysis. (a) Part of the USAF test image. (b) Image obtained with CCD camera. (c) Reconstructed image of $128 \times 128$ pixels via single-pixel imaging $(\mathrm{CR}=2)$. Panels $(\mathrm{d})$ and (e) show intensity profiles for USAF elements 6-6 and 7-5, respectively. Blue lines correspond to the CCD camera and red lines to the single-pixel image. The curves have been smoothed. limited by diffraction to $\delta x=0.61 \lambda / \mathrm{NA}=3.33 \mu \mathrm{m}$ because of the low NA objective O2. In fact, as shown in Fig. 2(b), the conventional image provided by the CCD allows us to distinguish up to the element $6-5$ of the USAF test, corresponding to a resolution of $4.9 \mu \mathrm{m}$. This is also shown in blue profiles in Figs. 2(d) and 2(e) that correspond to elements $6-6$ and 7-5. However, as can be seen in Fig. 2(c), the resolution shown by our single-pixel configuration allows us to distinguish the element 7-5 of the USAF test, corresponding to a resolution limit of $2.46 \mu \mathrm{m}$. This fact is clearly noticeable in the red plots in Figs. 2(d) and 2(e), which allow us to discriminate the lines in the elements $6-6$ and 7-5 of the test. Therefore, the improvement of resolution with our single-pixel system is almost two times better than that provided by the conventional system. In Fig. 2(b) and more clearly in Fig. 2(c), a non-symmetry can also be observed between horizontal and vertical lines (CCD and single-pixel imaging respectively). This might be due to cylindrical aberration induced by the SLM. This aberration can be corrected but this is beyond the purpose of this work. These results show that the image obtained by computational imaging contains frequencies of the object that are above the frequency limit associated with the low-quality objective lens for conventional imaging. In other words, our single-pixel system is able to provide images with a resolution below the Rayleigh criterion. This is due to the spread of the spatial spectrum of the object produced by the projection of the high-frequency Hadamard patterns. The effect is similar to that produced by structured illumination in superresolution approaches based on spatial-frequency multiplexing [18].

To emphasize the fact that the resolution of the system does not depend on the collection system and is tolerant to some optical aberrations, next we prove that the system is able to recover images even when a diffuser distorts the light diffracted by the object. Figure 3(a) shows the image of the USAF test provided by the conventional imaging system when a commercial diffuser (D, Edmund Optics T54-497) is placed between the sample and the collection system. Note that the image is completely blurred by the action of the diffuser. Interestingly, the computational imaging approach based on single-pixel detection is able to reconstruct the image as can be seen in Fig. 3(b). In fact, the presence of the diffuser in the optical system in Fig. 1 can be advantageous because it improves the average ratio between low and high frequencies [19]. The combined action of the highfrequency patterns projected onto the object and the frequency mixture provided by the diffuser allow us to

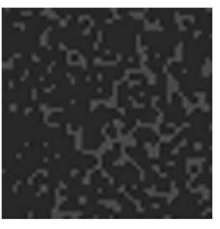

a

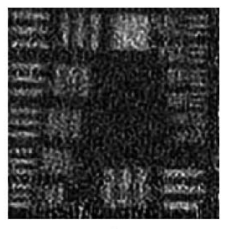

b
Fig. 3. Images taken through a commercial diffuser placed between $\mathrm{OB}$ and $\mathrm{O} 2$ (see Fig. 1). (a) Image obtained with CCD camera. (b) Reconstructed image via single-pixel imaging $(\mathrm{CR}=2)$. 
record frequencies of the object not resolved by conventional imaging systems.

The total time required to take image data increases with the number of measurements. The exact number $M$ of measurements that allows to provide an accurate reconstruction of an object from an undersampled signal is not a priori known. In addition, this number strongly depends on the features of the object under consideration. For this reason, we tested the quality of the recovered images evaluating the standard peak signal-to-noise ratio $(P S N R)$ and the acquisition time for different values of $M$. When $M=8192(\mathrm{CR}=2)$, it exists as a trade-off between image quality and acquisition time because the time required for the sensing stage could be reduced by a factor of 2 while the PSNR is still higher than $20 \mathrm{~dB}$, which indicates high image fidelity. In our experimental setup, the PMT can detect light signals at frequencies as high as $20 \mathrm{kHz}$. However, the acquisition frequency is limited to $60 \mathrm{~Hz}$ by the frame rate of the liquid crystal SLM. Furthermore, this kind of SLM provides amplitude values with slight temporal fluctuations, which are causing the noise in our experimental results in Figs. 2(c) and 3(b). These points can be easily improved by using DMD modulators, which can operate at much higher rates [7] and with better stability.

In conclusion, we have demonstrated that single-pixel imaging improves resolution of conventional imaging systems overcoming the Rayleigh criterion. This is due to the use of high-frequency Hadamard patterns in the projection system but also to the blindness of the collection system to optical aberrations. This blindness goes so far that we are able to reconstruct an image even when the light has been transmitted through a diffusing medium. The use of a PMT also allows imaging with very low light levels (a common situation in fluorescence microscopy) and produces an increase in signal-to-noise ratio due to the gain of this type of sensor. However, single-pixel imaging is not free from drawbacks, like the increase in acquisition time, limited in our case by the SLM sample rate.

This work was supported by the Generalitat Valenciana through projects PROMETEO/2012/021, ISIC/2012/
013, and by the Universitat Jaume I through project P1-1B2012-55. A. D. Rodríguez acknowledges grant PREDOC/2012/41 from Universitat Jaume I.

\section{References}

1. J. H. Shapiro, Phys. Rev. A 78, 061802 (2008).

2. B. I. Erkmen and J. H. Shapiro, Adv. Opt. Photon. 2, 405 (2010).

3. M. F. Duarte, M. A. Davenport, D. Takhar, J. N. Laska, K. F. Kelly, and R. G. Baraniuk, IEEE Signal Process. Mag. 25(2), 83 (2008).

4. V. Studer, J. Bobin, M. Chahid, H. S. Mousavi, E. Candes, and M. Dahan, Proc. Natl. Acad. Sci. USA 109, E1679 (2012).

5. F. Soldevila, E. Irles, V. Durán, P. Clemente, M. FernándezAlonso, E. Tajahuerce, and J. Lancis, Appl. Phys. B 113, 551 (2013).

6. V. Durán, P. Clemente, M. Fernández-Alonso, E. Tajahuerce, and J. Lancis, Opt. Lett. 37, 824 (2012).

7. G. A. Howland, D. J. Lum, M. R. Ware, and J. C. Howell, Opt. Express 21, 23822 (2013).

8. B. M. Davis, A. J. Hemphill, D. Cebeci Maltaş, M. A. Zipper, P. Wang, and D. Ben-Amotz, Anal. Chem. 83, 5086 (2011).

9. E. J. Candes and M. B. Wakin, IEEE Signal Process. Mag. 25 (5), 682 (2008).

10. J.-E. Oh, Y.-W. Cho, G. Scarcelli, and Y.-H. Kim, Opt. Lett. 38, 682 (2013).

11. W. Gong, Z. Bo, E. Li, and S. Han, Appl. Opt. 52, 3510 (2013).

12. A. Szameit, Y. Shechtman, E. Osherovich, E. Bullkich, P. Sidorenko, H. Dana, S. Steiner, E. B. Kley, S. Gazit, T. Cohen-Hyams, S. Shoham, M. Zibulevsky, I. Yavneh, Y. C. Eldar, O. Cohen, and M. Segev, Nat. Mater. 11, 455 (2012).

13. R.-W. Lu, B.-Q. Wang, Q.-X. Zhang, and X.-C. Yao, Biomed. Opt. Express 4, 1673 (2013).

14. T. Shirai, H. Kellock, T. Setälä, and A. T. Friberg, J. Opt. Soc. Am. A 29, 1288 (2012).

15. A. P. Mosk, A. Lagendijk, G. Lerosey, and M. Fink, Nat. Photonics 6, 283 (2012).

16. E. J. Candes, http://users.ece.gatech.edu/justin/11magic.

17. T. Wilson, Theory and Practice of Scanning Optical Microscopy (Academic, 1984).

18. Z. Zalevsky and D. Mendlovic, Optical Superresolution (Springer, 2003).

19. Y. Choi, T. D. Yang, C. Fang-Yen, P. Kang, K. J. Lee, R. R. Dasari, M. S. Feld, and W. Choi, Phys. Rev. Lett. 107, 023902 (2011). 